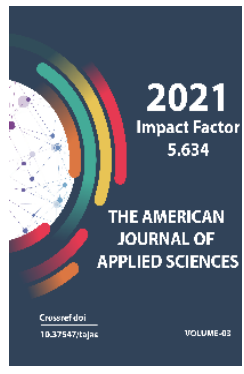

\title{
Kompl Eyler's Formula In Hex Analysis And Some Results In Elementary And Higher Mathematics
}

\author{
Sulaymonov Ilyosxo'ja Abdirasul O'g'li \\ 2nd Year Bachelor Student Of The Faculty Of Applied Mathematics And Intellectual \\ Technologies Of The National University Of Uzbekistan Named After Mirzo Ulugbek, \\ Uzbekistan
}

Journal Website:

http://usajournalshub.c

om/index,php/tajas

Copyright: Original

content from this work

may be used under the

terms of the creative

commons attributes

4.0 licence.

\section{ABSTRACT}

This article is devoted to solving some of the problems of elementary mathematics with the help of a conformal invariant, which has its own advantages, applied in the theory of modern functions, as well as some important issues of mathematical analysis with the help of Komplex numbers, several results obtained with the help of the Eyler formula and their application in practice.

\section{KEYWORDS}

$z_{n}, \quad z_{n}=\lim _{n \rightarrow \infty}\left(1+\frac{z}{n}\right)^{n}$, Eyler formula, aggregate, integral, $\sin x=\frac{1}{2 i}\left(e^{i x}-e^{-i x}\right)$, $\cos x=\frac{1}{2}\left(e^{i x}+e^{-i x}\right)$ 


\section{INTRODUCTION}

1. Eyler formula

2. Kompl let's look at the following sequence by taking an optional $z$ number in the plane of hexadecimal numbers

3.

$$
z_{n}=\left(1+\frac{z}{n}\right)^{n}
$$

4.It is known that Komplex sequence of numbers $n \rightarrow \infty$ has a limit.

5.From the course of mathematical analysis we know that this $z_{n}=\lim _{n \rightarrow \infty}\left(1+\frac{z}{n}\right)^{n}$

6.sequence limit $e^{z}$ the function is called.

$z \in C$ Komplex number this $z=r(\cos \varphi+i \sin \varphi)$ given in appearance $\mathrm{n}$. Komplex is one of the following important formulas known from the course of analysis

$$
e^{i \varphi}=\cos \varphi+i \sin \varphi
$$

With the help of the Eyler formula Komplex number we can write the following

$$
z=r e^{i \varphi}
$$

We know that given two $z_{1}=x_{1}+i y_{1}$ va $z_{2}=x_{2}+i y_{2}$ Komplex numbers to be equal $x_{1}=x_{2}$ va $y_{1}=y_{2}$ be necessary and sufficient

Let's go through the solutions of the results obtained using the Eyler formula and a few examples that are important.

\section{Results obtained using Eyler formula} 1-example. Calculate the given sum

a) $1+\cos x+\cos 2 x+\ldots+\cos n x$

b) $\sin x+\sin 2 x+\ldots+\sin n x$

$S_{1}=1+\cos x+\cos 2 x+\ldots+\cos n x$ and $S_{2}=\sin x+\sin 2 x+\ldots+\sin n x$ let it be. Let's look at the sum as follows

$$
S_{1}+i S_{2}=1+\cos x+i \sin x+\cos 2 x+i \sin 2 x+\ldots+\cos n x+i \sin n x
$$


According to Eyler's formula, we can write this sum as follows

$$
\begin{aligned}
& S_{1}+i S_{2}=1+e^{i x}+e^{i 2 x}+\ldots+e^{i n x}=\frac{e^{i(n+1) x}-1}{e^{i x}-1}=\frac{\cos (n+1) x+i \sin (n+1) x-1}{(\cos x-1)+i \sin x}= \\
& =\frac{(\cos (n+1) x+i \sin (n+1) x-1)((\cos x-1)-i \sin x)}{(\cos x-1)^{2}+\sin ^{2} x}
\end{aligned}
$$

As a result of simplification of the formed expression, we get the following

$$
\begin{aligned}
& S_{1}+i S_{2}=\frac{\cos (n x)-\cos ((n+1) x)-\cos (x)+1}{2-2 \cos x}+ \\
& +i \frac{\sin (n x)-\sin ((n+1) x)+\sin (x)}{2-2 \cos x}
\end{aligned}
$$

Komplex according to the theorem on the equality of numbers

$$
\begin{aligned}
& S_{1}=\frac{\cos (n x)-\cos ((n+1) x)-\cos (x)+1}{2-2 \cos x} \\
& S_{2}=\frac{\sin (n x)-\sin ((n+1) x)+\sin (x)}{2-2 \cos x}
\end{aligned}
$$

2-example. Calculate the sum given below

$$
S_{n}=\cos x+2 \cos 2 x+3 \cos 3 x+\ldots+n \cos n x
$$

Solution Initially, we calculate the following sum

$$
T_{n}=\sin x+\sin 2 x+\sin 3 x+\ldots+\sin n x
$$

Through the above examples, it is known that the sum is equal to

$$
T_{n}=\frac{\sin n x-\sin (n+1) x+\sin x}{2-2 \cos x}
$$

From what is known, the first orderly harvest will be equal to. All in all 


$$
\begin{aligned}
& S_{n}=\left(\frac{\sin n x-\sin (n+1) x+\sin x}{2-2 \cos x}\right)^{\prime}=\left(\frac{\sin \left(\frac{n x}{2}\right) \sin \left(\frac{n+1}{2} x\right)}{\sin \left(\frac{x}{2}\right)}\right)^{\prime}= \\
& =\frac{n \sin \frac{x}{2} \sin \left(\frac{2 n+1}{2} x\right)-\sin ^{2} \frac{n x}{2}}{2 \sin ^{2} \frac{x}{2}}
\end{aligned}
$$

3- example. Calculate:

$$
\begin{aligned}
& I_{1}=\int e^{a x} \cos (b x) d x \\
& I_{2}=\int e^{a x} \sin (b x) d x
\end{aligned}
$$

Solution: we write the above two integers as follows

$$
I_{1}+i I_{2}=\int e^{a x} \cos (b x) d x+i \int e^{a x} \sin (b x) d x
$$

We look at the integral below.

$$
\begin{aligned}
& \int e^{a x} e^{i b x} d x=\int e^{(a+i b) x} d x=\frac{e^{(a+i b) x}}{a+i b}+C=\frac{a-i b}{a^{2}+b^{2}} e^{(a+i b) x}+C= \\
& =\frac{a-i b}{a^{2}+b^{2}} e^{a x} e^{i b x}+C
\end{aligned}
$$

Now according to Eyler's formula $e^{i b x}=\cos b x+i \sin b x$ we can write the above example as follows

$$
\frac{(a-i b)(\cos b x+i \sin b x)}{a^{2}+b^{2}} e^{a x}=\frac{a \cos b x+b \sin b x}{a^{2}+b^{2}} e^{a x}+i \frac{a \sin b x-b \cos b x}{a^{2}+b^{2}} e^{a x}
$$

As a result of the equation of (1) and (2) expressions according to the above theorem, we get the following

$$
\begin{aligned}
& \int e^{a x} \cos (b x) d x=\frac{a \cos b x+b \sin b x}{a^{2}+b^{2}} e^{a x}+C \\
& \int e^{a x} \sin (b x) d x=\frac{a \sin b x-b \cos b x}{a^{2}+b^{2}} e^{a x}+C
\end{aligned}
$$




\section{4-example. Calculate:}

$\int_{0}^{\pi} \frac{\sin n x}{\sin x} d x$

Solution: according to the known Eyler formula $\sin x=\frac{1}{2 i}\left(e^{i x}-e^{-i x}\right)$ and

$\sin n x=\frac{1}{2 i}\left(e^{i n x}-e^{-i n x}\right)$ all in all

$\frac{\sin n x}{\sin x}=\frac{e^{i n x}-e^{-i n x}}{e^{i x}-e^{-i x}}=\sum_{k=1}^{n} e^{i(n+1-2 k) x}=e^{i(n-1) x}+e^{i(n-3) x}+\ldots+e^{-i(n-3) x}+e^{-i(n-1) x}(1)$

$\cos x=\frac{1}{2}\left(e^{i x}+e^{-i x}\right)$ given that (1) we can write the expression as follows

$e^{i(n-1) x}+e^{i(n-3) x}+\ldots+e^{-i(n-3) x}+e^{-i(n-1) x}=$
$=\left\{\begin{array}{l}2(\cos (n-1) x+\cos (n-3) x+\ldots+\cos x), n-j u f t \\ 2(\cos (n-1) x+\cos (n-3) x+\ldots \cos x)+1, n-t o q\end{array}\right.$

Now we look at the following integral

$\int_{0}^{\pi} \cos (n-k) x d x=\left.\frac{\sin (n-k) x}{n-k}\right|_{0} ^{\pi}=0$

Hence (3) according to the expression

$$
\int_{0}^{\pi} \frac{\sin n x}{\sin x} d x=\left\{\begin{array}{l}
0, n-j u f t \\
\pi, n-t o q
\end{array}\right.
$$

5-example. Calculate:

$$
I=\int_{0}^{\pi} \frac{\cos ((2 n+1) x)}{\cos x} d x
$$

Solution: according to Eyler formula $\cos x=\frac{1}{2}\left(e^{i x}+e^{-i x}\right)$ furthermore 


$$
\begin{aligned}
& \frac{\cos ((2 n+1) x)}{\cos x}=\frac{e^{i(2 n+1) x}+e^{-i(2 n+1) x}}{e^{i x}+e^{-i x}}=e^{i 2 n x}-e^{i(2 n-2) x}+\ldots+e^{-i 2 n x}= \\
& =2 \sum_{k=1}^{n}(-1)^{k-1} \cos (2(n-(k-1)) x)+(-1)^{n}
\end{aligned}
$$

(1) through the expression, we can write the above given integral as follows

$$
\begin{aligned}
& I=2 \sum_{k=1}^{n} \int_{0}^{\pi} \cos (2(n-(k-1)) x) d x+(-1)^{n} \pi=\left.2 \sum_{k=1}^{n} \frac{\sin (2(n-(k-1)) x)}{2(n-(k-1))}\right|_{0} ^{\pi}+(-1)^{n} \pi= \\
& =(-1)^{n} \pi
\end{aligned}
$$

\section{6- example. Calculate:}

$$
\begin{aligned}
& \mathrm{S}_{1}=q \sin x+q^{2} \sin 2 x+q^{3} \sin 3 x+\ldots+q^{n} \sin n x+\ldots \\
& S_{2}=q \cos x+q^{2} \cos 2 x+q^{3} \cos 3 x+\ldots+q^{n} \cos n x+\ldots
\end{aligned}
$$

Solution. Let's look at the sum below

$$
S_{1}+i S_{2}=q(\cos x+i \sin x)+q^{2}(\cos 2 x+i \sin 2 x)+\ldots+q^{n}(\cos n x+i \sin n x)+\ldots
$$

According to Eyler's formula

$$
S_{1}+i S_{2}=q e^{i x}+q^{2} e^{i x}+\ldots+q^{n} e^{i n x}+\ldots=\frac{q e^{i x}}{1-q e^{i x}}=\frac{q(\cos x+i \sin x)}{1-q \cos x-i q \sin x}
$$

As a result of simplifying this generated sum, we will have the following

$$
S_{1}+i S_{2}=\frac{q \cos x-q^{2}}{1-2 q \cos x+q^{2}}+i \frac{q \sin x}{1-2 q \cos x+q^{2}}
$$

According to the theorem about the equality of commas numbers

$$
\begin{aligned}
& S_{1}=\frac{q \cos x-q^{2}}{1-2 q \cos x+q^{2}} \\
& S_{2}=\frac{q \sin x}{1-2 q \cos x+q^{2}}
\end{aligned}
$$




\section{REFERENCES}

1. Godberganov G., Verisov A., Mansurov H. Eksex analysis.Tashkent, University, 1998

2. Fichtenholz G. Course of differential and integral calculus. Vol. Il. Moscow, Физ Fizmat-lit》, 2001

3. Demidovich B. Collection of problems and exercises in mathematical analysis. Moscow, Наука Nauka》, 1990 